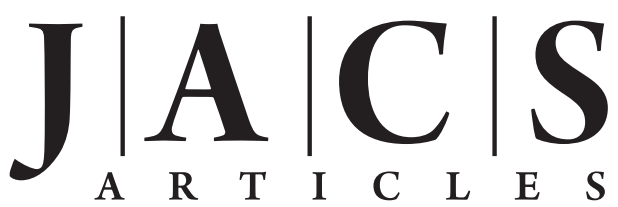

Published on Web 02/17/2010

\title{
Mechanism and Kinetics of Peptide Partitioning into Membranes from All-Atom Simulations of Thermostable Peptides
}

\author{
Martin B. Ulmschneider, ${ }^{*, \dagger}$ Jacques P. F. Doux, ${ }^{\dagger}$ J. Antoinette Killian, ${ }^{\dagger}$ \\ Jeremy C. Smith, ${ }^{\ddagger, \S}$ and Jakob P. Ulmschneider ${ }^{*, \S}$ \\ Department of Chemistry, University of Utrecht, Utrecht, The Netherlands, Oak Ridge National \\ Laboratory, Oak Ridge, Tennesee, and IWR, University of Heidelberg, Germany
}

Received November 4, 2009; E-mail: martin@ulmschneider.com; jakob@ulmschneider.com

\begin{abstract}
Partitioning properties of transmembrane (TM) polypeptide segments directly determine membrane protein folding, stability, and function, and their understanding is vital for rational design of membrane active peptides. However, direct determination of water-to-bilayer transfer of TM peptides has proved difficult. Experimentally, sufficiently hydrophobic peptides tend to aggregate, while atomistic computer simulations at physiological temperatures cannot yet reach the long time scales required to capture partitioning. Elevating temperatures to accelerate the dynamics has been avoided, as this was thought to lead to rapid denaturing. However, we show here that model TM peptides (WALP) are exceptionally thermostable. Circular dichroism experiments reveal that the peptides remain inserted into the lipid bilayer and are fully helical, even at 90 ${ }^{\circ} \mathrm{C}$. At these temperatures, sampling is $\sim 50-500$ times faster, sufficient to directly simulate spontaneous partitioning at atomic resolution. A folded insertion pathway is observed, consistent with three-stage partitioning theory. Elevated temperature simulation ensembles further allow the direct calculation of the insertion kinetics, which is found to be first-order for all systems. Insertion barriers are $\Delta H_{\text {in }}{ }^{\ddagger}=15 \mathrm{kcal} / \mathrm{mol}$ for a general hydrophobic peptide and $\sim 23 \mathrm{kcal} / \mathrm{mol}$ for the tryptophan-flanked WALP peptides. The corresponding insertion times at room temperature range from $8.5 \mu \mathrm{s}$ to $163 \mathrm{~ms}$. High-temperature simulations of experimentally validated thermostable systems suggest a new avenue for systematic exploration of peptide partitioning properties.
\end{abstract}

\section{Introduction}

Determination of the partitioning properties of polypeptide segments into lipid bilayers is critical to understanding the insertion, assembly, and function of membrane proteins. ${ }^{1}$ Unfortunately, experiments aimed at directly measuring the transfer of peptides from water into bilayers have not so far been successful, primarily because sequences that are sufficiently hydrophobic to spontaneously insert without disrupting the membrane have a tendency to aggregate. ${ }^{2,3}$ Current partitioning theory has instead been assembled from a large body of indirect experimental and computational evidence, as previously reviewed. ${ }^{4,5}$ The physical basis is the trans-bilayer hydrophobicity profile, which allows suitably hydrophobic peptides to bury themselves through helix formation. Unfolded insertion is generally ruled out due to the large energetic penalty associated with desolvating an exposed peptide backbone. ${ }^{5}$ This vital backbone contribution is lacking in transmembrane potentials

$\dagger$ University of Utrecht.

$\doteqdot$ Oak Ridge National Laboratory.

${ }^{\S}$ University of Heidelberg.

(1) White, S. H.; von Heijne, G. Annu. Rev. Biophys. 2008, 37, 23-42.

(2) Wimley, W. C.; White, S. H. Biochemistry 2000, 39 (15), 4432-42.

(3) Ladokhin, A. S.; White, S. H. Biochemistry 2004, 43 (19), 57825791.

(4) White, S. H.; Wimley, W. C. Annu. Rev. Biophys. Biomol. Struct. 1999, 28, 319-365.

(5) White, S. H. Adv. Protein Chem. 2006, 72, 157-172. of mean force derived for side chain analogs ${ }^{6}$ or small peptides that cannot assume secondary structure. ${ }^{7}$ Computational methods in which the membrane is represented implicitly have been developed and applied to simulate folding and insertion of small hydrophobic peptides, providing a confirmation of the folded insertion pathway and rough estimates of the energetics involved. ${ }^{8-10}$ However, the complex physiochemical properties of fluid phase lipid bilayers, especially at the interfaces, have proved difficult to capture with implicit solvation models.

Clearly, an atomic detail bilayer description is desirable. However, previous molecular dynamics simulations studying the partitioning of WALP peptides into explicit DPPC bilayers have reported incorrect insertion pathways due to inadequate simulation time scales and problems with the underlying lipid parameters. ${ }^{11,12}$ Crucial to the success of the present project was a new lipid parametrization recently carried out by us. ${ }^{13}$ We present here an approach that determines the monomeric partitioning properties of hydrophobic peptides into lipid bilayers

(6) MacCallum, J. L.; Bennett, W. F. D.; Tieleman, D. P. J. Gen. Physiol. 2007, 129 (5), 371-377.

(7) Babakhani, A.; Gorfe, A. A.; Kim, J. E.; McCammon, J. A. J. Phys. Chem. B 2008, 112 (34), 10528-34.

(8) Im, W.; Brooks, C. L. Proc. Natl. Acad. Sci. U.S.A. 2005, 102 (19), 6771-6776.

(9) Ulmschneider, M. B.; Ulmschneider, J. P. Mol. Membr. Biol. 2008, 25 (3), 245-257.

(10) Ulmschneider, J. P.; Ulmschneider, M. B. J. Chem. Theory Comput. 2007, 3, 2335-2346. 
through all-atom simulation at elevated temperatures. Key is the remarkable stability against thermal denaturation of hydrophobic membrane inserting peptides, validated using circular dichroism experiments. This enables simple direct determination of atomic detail information on the partitioning pathways, intermediate states, as well as insertion kinetics and related barriers.

\section{Materials and Methods}

Molecular Dynamics. Simulations were performed and analyzed using Gromacs version 4.0 (www.gromacs.org) ${ }^{14}$ and Hippo BETA (www.biowerkzeug.com). All systems were simulated in the NPT ensemble using the OPLS all-atom (OPLS-AA) protein force field ${ }^{15}$ in combination with the TIP3P water model ${ }^{16}$ and OPLS-AAcompatible united-atom lipid parameters recently parametrized by us. ${ }^{13}$ Electrostatic interactions were computed using PME, and a cutoff of $10 \AA$ was used for van der Waals interactions. Bonds involving hydrogen atoms were restrained using LINCS. ${ }^{17}$ Simulations were run with a 2 fs integration time step and neighbor lists were updated every five steps. Water, lipids, and the protein were each coupled separately to a heat bath with time constant $\tau_{\mathrm{T}}=0.1$ ps using weak temperature coupling. ${ }^{18}$ Atmospheric pressure of 1 bar was maintained using weak semi-isotropic pressure coupling with compressibility $\kappa_{z}=\kappa_{x y}=4.6 \times 10^{-5} \mathrm{bar}^{-1}$ and time constant $\tau_{\mathrm{P}}=1$ ps. $^{19}$

Control Simulations. We have previously established that the OPLS-AA force field used in our simulations has no underlying helical bias. ${ }^{20}$ For the present study further control simulations were performed to ascertain that peptide folding is a consequence of partitioning into the hydrophobic lipid bilayer and not an intrinsic property of the peptide sequence itself.

This issue was addressed by simulating equilibrated helical conformers in water. The helices unfold immediately (>70\% loss of helicity in $<100 \mathrm{~ns})$ and do not refold $(<10 \%$ helicity over 500 ns) at both 27 and $80{ }^{\circ} \mathrm{C}$, demonstrating that folding is a result of the membrane environment rather than an intrinsic property of the sequence or a helical bias in the OPLS-AA force field.

Bilayer Mismatch Energetics. The perturbation of the bilayer surface around the transmembrane-inserted peptide was quantified using a single-order parameter model based on Landau-de Gennes theory. ${ }^{21}$ Within this theoretical framework, the free energy of the peptide-induced membrane deformations is calculated by minimizing a functional integral over the local free energy density. The order parameter is taken to be the local hydrophobic membrane thickness $d_{\mathrm{L}}(r)$ (calculated as the distance of the phosphate groups, which is proportional) as a function of radial distance $r$ from the center of mass of the peptide. For elastic bilayer deformations and harmonic local thickness fluctuations, this predicts an exponential

(11) Nymeyer, H.; Woolf, T. B.; Garcia, A. E. Proteins: Struct. Funct. Bioinf. 2005, 59 (4), 783-790.

(12) Ulmschneider, M. B.; Ulmschneider, J. P. J. Chem. Theory Comput. 2008, 4, 1807-1809.

(13) Ulmschneider, J. P.; Ulmschneider, M. B. J. Chem. Theory Comput. 2009, 5 (7), 1803-1813.

(14) Berendsen, H. J. C.; van der Spoel, D.; van Drunen, R. Comput. Phys. Commun. 1995, 95, 43-56.

(15) Jorgensen, W. L.; Maxwell, D. S.; Tirado-Rives, J. J. Am. Chem. Soc. 1996, 118 (45), 11225-11236.

(16) Jorgensen, W. L.; Chandrasekhar, J.; Madura, J. D.; Impey, R. W.; Klein, M. L. J. Chem. Phys. 1983, 79 (2), 926-935.

(17) Hess, B.; Bekker, H.; Berendsen, H. J. C.; Fraaije, J. G. E. M. J. Comput. Chem. 1997, 18, 1463-1472.

(18) Bussi, G.; Donadio, D.; Parrinello, M. J. Chem. Phys. 2007, 126 (1), 014101.

(19) Berendsen, H. J. C.; Postma, J. P. M.; Vangunsteren, W. F.; Dinola, A.; Haak, J. R. J. Chem. Phys. 1984, 81 (8), 3684-3690.

(20) Ulmschneider, J. P.; Ulmschneider, M. B.; Di Nola, A. Proteins: Struct., Funct., Bioinf. 2007, 69, 297-308.

(21) Jensen, M. O.; Mouritsen, O. G. Biochim. Biophys. Acta 2004, 1666 (1-2), 205-26. surface shape linking the hydrophobic widths of the peptide and the bilayer at infinite distance from the peptide:

$$
d_{\mathrm{L}}(r)=d_{\mathrm{P}}-\left(d_{\mathrm{L}}^{\infty}-d_{\mathrm{P}}\right) \mathrm{e}^{-r / \xi}
$$

where $d_{\mathrm{P}}$ is the hydrophobic width of the peptide, $d_{\mathrm{L}}^{\infty}$ is the unperturbed hydrophobic thickness of the bilayer (far from the peptide), and $\xi$ is a deformation coherence length for the perturbation, which can be related to the bilayer elastic properties. Fitting the equilibrium surface perturbation $d_{\mathrm{L}}(r)$ to eq 1 yields $d_{\mathrm{P}}$ and $\xi$. $d_{\mathrm{L}}^{\infty}$ can be obtained from pure bilayer simulations. This allows one to estimate the relative free energy contribution associated with peptide-lipid interactions via

$$
\Delta G=k\left(\frac{2 R}{\xi}+1\right)\left(d_{\mathrm{L}}^{\infty}-d_{\mathrm{P}}\right)^{2}
$$

where $R$ is the radius of the peptide $(\sim 5 \AA)$ and $k$ is a phenomenological constant.

Sample Preparation. Dimyristoylphosphatidylcholine (DMPC), dipalmitoylphosphatidylcholine (DPPC) and dioleoylphosphatidylcholine (DOPC) lipids were obtained from Avanti Polar Lipids (Alabaster, AL). Acetylated (ace) and amidated (ame) W16 [aceAWW-(LA) ${ }_{5}$-WWA-ame] and W23 [ace-GWW-(LA) ${ }_{8}$ L-WWAame] peptides were solid-phase synthesized using Fmoc/HBTU chemistry and quantified using mass spectroscopy as described previously. ${ }^{22,23}$ Samples were prepared by mixing stock solutions of peptide in trifluoroethanol and lipids in chloroform. The mixture was dried under a stream of nitrogen with subsequent overnight incubation under vacuum to remove all solvent traces. Vesicles were prepared by rehydrating dry peptide/lipid films in $1 \mathrm{~mL}$ of distilled water at room temperature to reach a peptide concentration of 30 $\mu \mathrm{M}$. After 10 freeze - thawing cycles, the vesicles were extruded 10 times through $0.2 \mu \mathrm{m}$ filters. The peptide concentration in all samples was $30 \mu \mathrm{M}$, and the molar peptide/lipid ratio was varied between $1 / 25$ and $1 / 100$. WALP concentrations were quantified by the average absorbance of tryptophan at $280 \mathrm{~nm}$ using a molar extinction coefficient of $\varepsilon=4 \times 5600=22400 \mathrm{M}^{-1} \mathrm{~cm}^{-124}$ and absorption at $214 \mathrm{~nm}$ using $\varepsilon=137220 \mathrm{M}^{-1} \mathrm{~cm}^{-1} \cdot{ }^{25}$ Lipid concentration was determined by a phosphorus titration according to the method of Rouser. ${ }^{26}$

Circular Dichroism. Measurements were carried on a JASCO J-810 spectropolarimeter, using a $1 \mathrm{~mm}$ path length quartz cuvette, $1 \mathrm{~nm}$ bandwidth, $0.2 \mathrm{~nm}$ resolution, $1 \mathrm{~s}$ response time, and a scan speed of $20 \mathrm{~nm} / \mathrm{min}$. Temperature was controlled with a Peltier device and varied between 20 and $90{ }^{\circ} \mathrm{C}$ in $5^{\circ}$ steps. The sample was equilibrated for $5 \mathrm{~min}$ at each temperature before collecting data, and measurements were repeated five times. The averaged spectra were smoothed using a Fourier filter and normalized to molar ellipticity per residue. All spectra were corrected for background scattering by subtracting the corresponding vesicleonly spectrum measured over the same temperature range. Secondary structure content was quantified using $\operatorname{CDSSTR}^{27}$ and CONTINLL $^{28}$ as implemented in the CDpro software package ${ }^{29}$ following standard protocols. ${ }^{30}$

\section{Results}

Peptide Partitioning Pathways. We determined the insertion properties of three hydrophobic peptides (5L, W16, and W23)

(22) de Planque, M. R.; Kruijtzer, J. A.; Liskamp, R. M.; Marsh, D.; Greathouse, D. V.; Koeppe, R. E., 2nd; de Kruijff, B.; Killian, J. A. J. Biol. Chem. 1999, 274 (30), 20839-20846.

(23) de Planque, M. R. R.; Goormaghtigh, E.; Greathouse, D. V.; Koeppe, R. E.; Kruijtzer, J. A. W.; Liskamp, R. M. J.; de Kruijff, B.; Killian, J. A. Biochemistry 2001, 40 (16), 5000-5010.

(24) Pace, C. N.; Vajdos, F.; Fee, L.; Grimsley, G.; Gray, T. Protein Sci. 1995, 4 (11), 2411-23.

(25) Kuipers, B. J.; Gruppen, H. J. Agric. Food Chem. 2007, 55 (14), 544551.

(26) Rouser, G.; Fkeischer, S.; Yamamoto, A. Lipids 1970, 5 (5), 494-6.

(27) Johnson, W. C. Proteins: Struct., Funct., Genet. 1999, 35 (3), 307312 . 


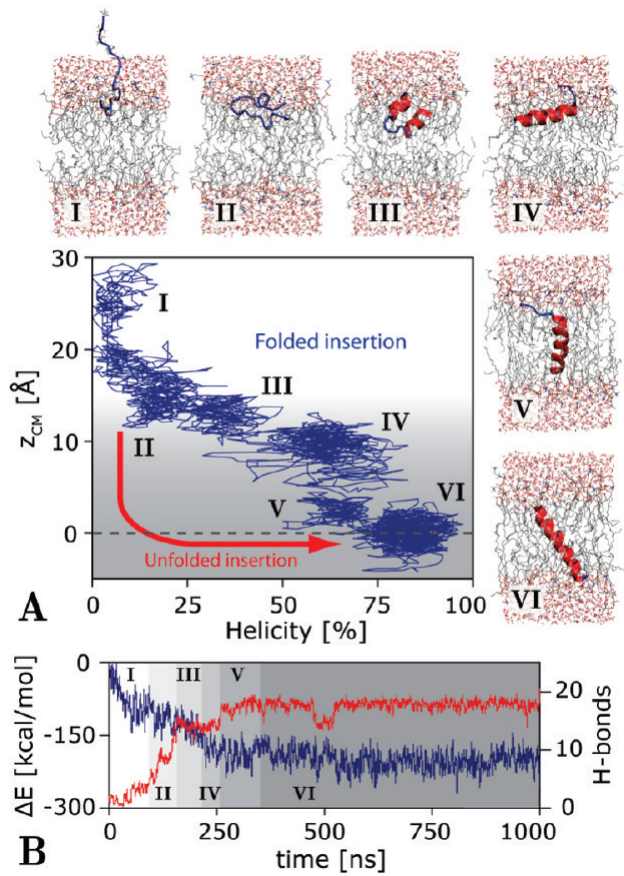

Figure 1. (A) Folding and insertion of the $5 \mathrm{~L}$ peptide at $80^{\circ} \mathrm{C}$. The peptide follows a folded insertion mechanism that can be visualized by plotting the center of mass $z$-position versus peptide helicity: Adsorption (I $\rightarrow$ II) is followed by interfacial folding (III $\rightarrow$ IV) and subsequent insertion (V, VI). An arrow indicates the hypothetical unfolded insertion pathway that was not observed. (B) The total potential energy (blue, left axis) and number of backbone hydrogen bonds (red, right axis) reveal that folding is rapid and irreversible. The lifetimes of the individual insertion phases are indicated.

into DPPC bilayers using direct all-atom molecular dynamics simulations. All peptides were acetylated (N-terminus) and amidated (C-terminus) to avoid electrostatic interactions with the lipid headgroups and were initially placed in bulk water 10 $\AA$ from the bilayer surface in an extended configuration.

We first investigated a purely hydrophobic peptide. 5L (ace$\mathrm{A}_{4}$ LALAALAALALA $_{4}$-ame) is from a series of synthetic peptides designed by White and von Heijne to explore sequence dependent peptide-bilayer insertion propensities ${ }^{31,32}$ and is preferentially inserted $\left(\Delta G_{\text {app }}=-1.1 \mathrm{kcal} / \mathrm{mol}\right)$ into microsomal membranes by the Sec61 translocon using an in vitro assay. ${ }^{33}$ The direct partitioning pathway observed in the present simulations is summarized in Figure 1 and is thought to be representative for simple hydrophobic peptides. ${ }^{4,5}$ Rapid precipitation to the membrane interface is followed by interfacial folding into a surface-bound (SB) helix, which subsequently inserts to span the bilayer. This process is completed in $<300 \mathrm{~ns}$ at $80^{\circ} \mathrm{C}$. Once established, the transmembrane (TM) helix does not unfold or exit from the bilayer again.

Next, we investigated two model TM peptides that contain interfacial Trp anchors. W16 (ace-AWW-(LA) $)_{5}$-WWA-ame) and

(28) van Stokkum, I. H.; Spoelder, H. J.; Bloemendal, M.; van Grondelle, R.; Groen, F. C. Anal. Biochem. 1990, 191 (1), 110-118.

(29) Sreerama, N.; Venyaminov, S. Y.; Woody, R. W. Anal. Biochem. 2000, 287 (2), 243-51.

(30) Greenfield, N. J. Nature Protocols 2006, 1 (6), 2876-2890.

(31) Hessa, T.; Kim, H.; Bihlmaier, K.; Lundin, C.; Boekel, J.; Andersson, H.; Nilsson, I.; White, S. H.; von Heijne, G. Nature 2005, 433 (7024), 377-81.

(32) Hessa, T.; Meindl-Beinker, N. M.; Bernsel, A.; Kim, H.; Sato, Y.; Lerch-Bader, M.; Nilsson, I.; White, S. H.; von Heijne, G. Nature 2007, 450 (7172), 1026-1030.

(33) Saaf, A.; Wallin, E.; von Heijne, G. Eur. J. Biochem. 1998, 251 (3), $821-9$.

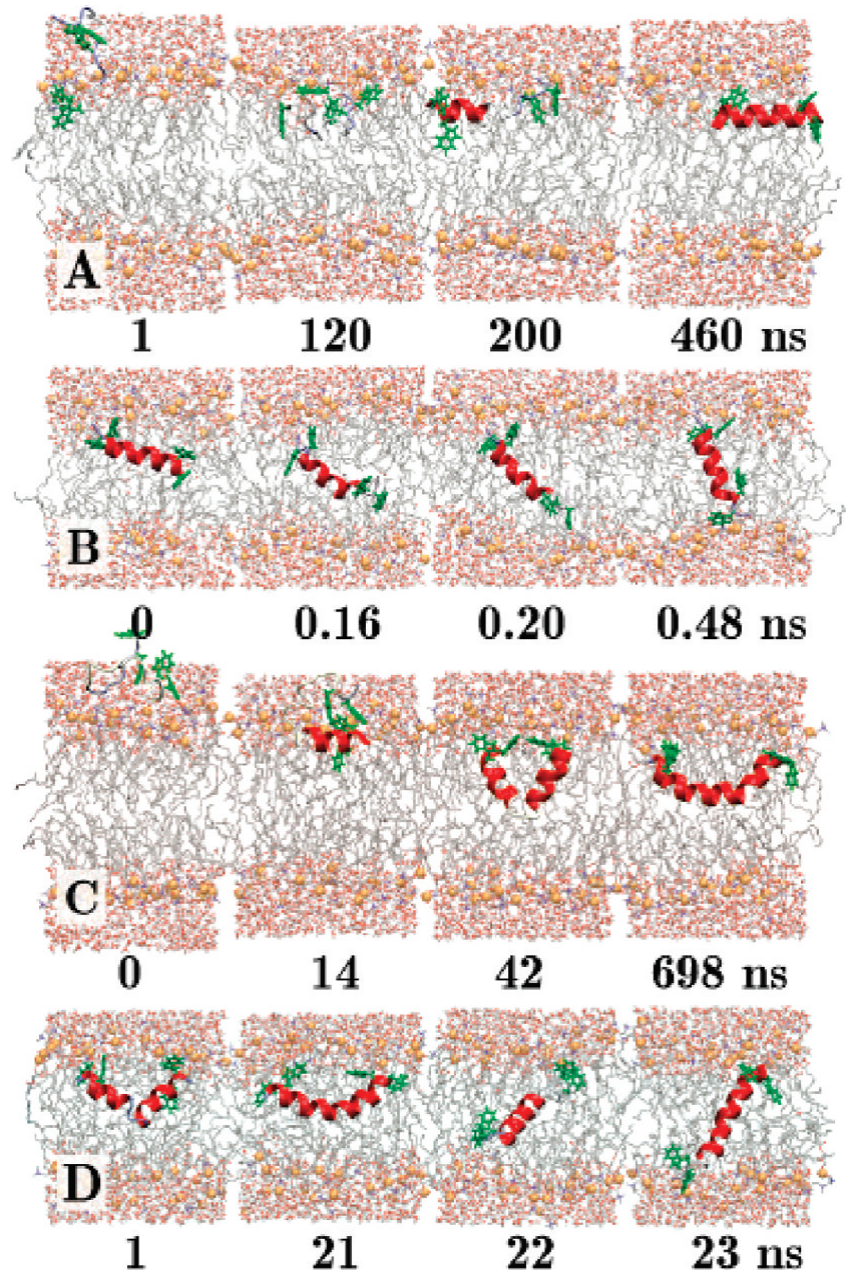

Figure 2. Peptide folding in DPPC bilayers at $80^{\circ} \mathrm{C}$. (A) Starting from extended conformations in water W16 quickly adsorbs to the bilayer interface and folds. The peptide remains kinetically trapped at the interface due to the high energetic cost of dissociating two tryptophan residues with the one interface and translocating them across the bilayer to the other. (B) The barrier is overcome by elevating the temperature to $200{ }^{\circ} \mathrm{C}$, making it thermally accessible. Similar results are obtained for W23 (C and D).

W23 (ace-GWW-(LA) ${ }_{8} \mathrm{~L}-\mathrm{WWA}-\mathrm{ame}$ ) are part of the WALP family of membrane-inserting peptides developed by Killian et al. to systematically explore peptide-bilayer interactions. ${ }^{34,35}$ For these peptides the biological hydrophobicity scale predicts larger insertion free energies of $\Delta G_{\text {app }}=-2.9$ (W16) and -5.0 $\mathrm{kcal} / \mathrm{mol}(\mathrm{W} 23){ }^{32}$ Simulation at $80{ }^{\circ} \mathrm{C}$ shows rapid adsorption and interfacial folding, but no insertion (Figure 2A,C). However, the peptides do insert when the temperature is elevated to 200 ${ }^{\circ} \mathrm{C}$ (Figure 2C,D). Clearly, the insertion barrier is much higher than for purely hydrophobic $5 \mathrm{~L}$.

Each peptide exhibits a characteristic interfacial state (SB): for $5 \mathrm{~L}$, this is a simple helix (Figure 3A). In contrast, the positions on the helical wheel of W16 mean that the formation of a perfect $\alpha$-helix at the interface would point at least one bulky Trp side chain into the bilayer core. This is energetically unfavorable, due to the strong interfacial anchoring of Trp residues. ${ }^{36} \mathrm{~W} 16$ compensates this mismatch by unwinding a

(34) Killian, J. A. FEBS Lett. 2003, 555 (1), 134-8.

(35) de Planque, M. R. R.; Killian, J. A. Mol. Membr. Biol. 2003, 20 (4), 271-284.

(36) Yau, W. M.; Wimley, W. C.; Gawrisch, K.; White, S. H. Biochemistry 1998, 37 (42), 14713-14718. 


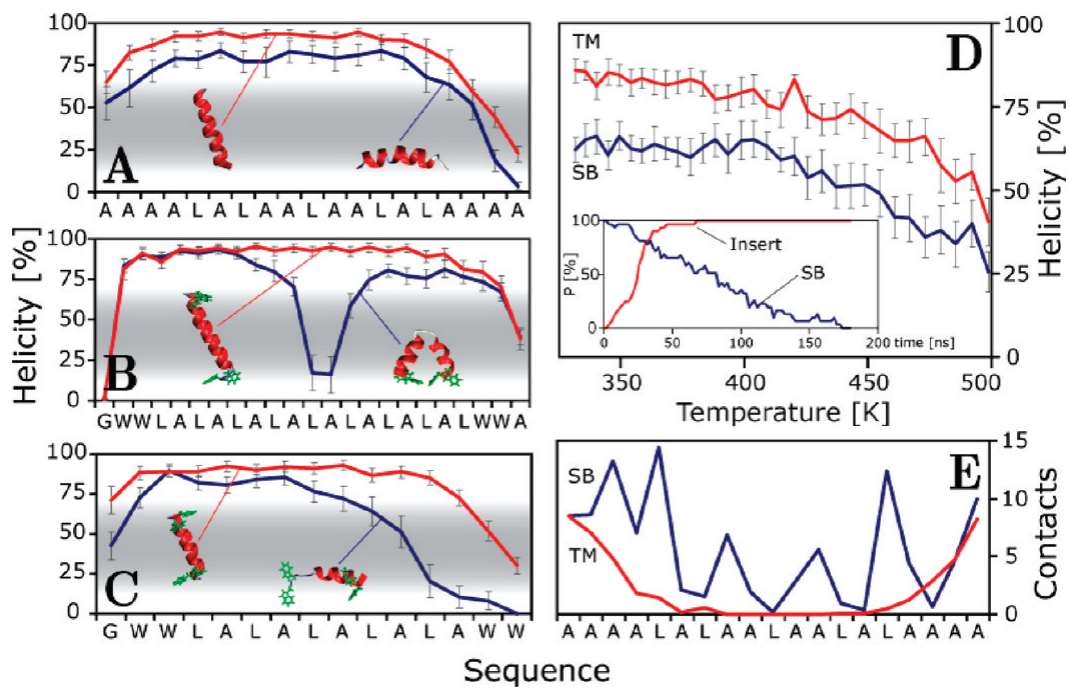

Figure 3. $(\mathrm{A}-\mathrm{C})$ Helicity of the surface-bound (SB, blue) and transmembrane (TM, red) states in the membrane. Representative configurations with respect to the hydrophobic membrane core (shaded) are indicated. $5 \mathrm{~L}$ forms a complete $\alpha$-helix at the surface (A). The WALP peptides adopt different ways to keep their terminal tryptophans at the interface, either by forming a turn region in the middle like W23 (B) or by terminal unwinding in the case of W16 (C). For all three peptides, the TM state is a fully formed $\alpha$-helix. REMD simulations of W16 reveal that the TM state is always more helical than the SB state over a wide temperature range (D). The number of replicas that have inserted at least once approaches $100 \%$ rapidly, but the SB state is observed to be only transitory, with the population averaged over all replicas declining to zero within $\sim 180 \mathrm{~ns}$ (D, inset). For all peptides, the TM states are much more stabilized than the SB states due to the better burial of hydrophobic surface, as can be seen from the number of water contacts for each residue (atom distance $<6 \AA$, $5 \mathrm{~L}$ peptide) (E).

Table 1. Thermodynamic and Kinetic Quantities of Partitioning of the Three Peptides Studied ${ }^{a}$

\begin{tabular}{lccc}
\hline & W16 & W23 & \multicolumn{1}{c}{$5 \mathrm{~L}$} \\
\hline$\Delta H_{\mathrm{U} \rightarrow \mathrm{SB}}$ & $-156 \pm 18$ & $-190 \pm 18$ & $-116 \pm 19$ \\
(per residue $)$ & $(-9.8 \pm 1.1)$ & $(-8.3 \pm 0.8)$ & $(-6.1 \pm 1)$ \\
$\Delta H_{\mathrm{SB} \rightarrow \mathrm{TM}}$ & $-12.0 \pm 9$ & $-23.6 \pm 8.5$ & $-40.7 \pm 8.9$ \\
$($ per residue $)$ & $(-0.8 \pm 0.9)$ & $(-1.0 \pm 0.7)$ & $(-2.1 \pm 0.5)$ \\
$\Delta H \operatorname{in}^{\ddagger}$ & $23.3 \pm 5$ & $24.2 \pm 6$ & $15.1 \pm 2$ \\
$-T \Delta \operatorname{Sin}^{\ddagger}\left(25^{\circ} \mathrm{C}\right)^{b}$ & $-12.6 \pm 4$ & $-13.1 \pm 4$ & $-9.9 \pm 3$ \\
$\Delta G \operatorname{in}^{\ddagger}\left(25^{\circ} \mathrm{C}\right)^{b}$ & $10.7 \pm 6$ & $11.0 \pm 7$ & $5.2 \pm 4$ \\
$k_{\text {in }}\left(25^{\circ} \mathrm{C}\right)$ & $9.3 \pm 1 \mathrm{~s}^{-1}$ & $6.1 \pm 0.9 \mathrm{~s}^{-1}$ & $0.1 \pm 0.03 \mu \mathrm{s}^{-1}$ \\
$\tau_{\text {in }}\left(25^{\circ} \mathrm{C}\right)$ & $107 \pm 15 \mathrm{~ms}$ & $163 \pm 24 \mathrm{~ms}$ & $8.5 \pm 3 \mu \mathrm{s}$ \\
$\tau_{\text {in }}\left(80^{\circ} \mathrm{C}\right)$ & $236 \pm 40 \mu \mathrm{s}$ & $287 \pm 35 \mu \mathrm{s}$ & $169 \pm 65 \mathrm{~ns}^{b}$ \\
$\tau_{\text {in }}\left(200^{\circ} \mathrm{C}\right)$ & $51 \pm 10 \mathrm{~ns}^{b}$ & $54 \pm 5 \mathrm{~ns}^{b}$ & $0.7 \pm 0.4 \mathrm{~ns}$
\end{tabular}

${ }^{a}$ All energies in $\mathrm{kcal} / \mathrm{mol} . \mathrm{U}=$ unfolded state, $\mathrm{SB}=$ surface-bound helix, $\mathrm{TM}=$ transmembrane helix. ${ }^{b}$ Direct measurements, other values from linear extrapolation of the Arrhenius plot.

helical turn at the C-terminus (Figure 3C). The longer W23 adopts a helix-turn-helix motif that fully inserts into one bilayer leaflet (Figure 3B). For all three peptides, the formation of the SB state results in a large enthalpy drop of $\Delta H_{\mathrm{U} \rightarrow \mathrm{SB}}=$ $\sim 6-10 \mathrm{kcal} / \mathrm{mol}$ per residue (Table 1, Figure 1B), concomitant with the burial of the hydrophobic peptide surface in the membrane and the formation of backbone hydrogen bonds.

Peptide Location and Bilayer Deformations. Where does the peptide localize and how does partitioning affect the accommodating bilayer? Figure 4A shows that the interfacial W16 helix is localized at a well-defined region $\left(z_{\mathrm{com}}=10.0 \pm 1.5\right.$ $\AA$ ) with respect to the principal structural groups of the bilayer corresponding to the alkane tail $\left(\mathrm{CH}_{2}\right)$-water interface, just below the phosphocholine $(\mathrm{P} / \mathrm{C})$ headgroups and carbonylglycerol $(\mathrm{C} / \mathrm{G})$ linker. Deeper penetration $\left(z_{\mathrm{com}}=5.8 \pm 2.2 \AA\right)$ is observed for the helix - turn-helix motif of W23 (Figure 5). This contrasts with amphiphilic peptides that locate in the semiaqueous part of the interface close to the glycerol linker $\left(z_{\text {com }} \sim 17 \AA\right) .{ }^{37,38}$ The surface deformation can be visualized by plotting the time-averaged phosphate elevation as a function of radial distance from the peptide's center of mass (Figure 4B). Local thinning in the vicinity of the peptide is caused by the headgroups bending over the helix in order to compensate for the bilayer expansion $(\sim 2 \%$ for a peptide/lipid ratio of $1 / 50)$ caused by the peptide (Figure 4B). W23 behaves similarly (Figure 5).

Once inserted in a TM configuration the headgroups must cover the extra area of the W16 helix leading to a circular decrease in bilayer width around the peptide (Figure 4B), consistent with a negatively mismatched peptide. For elastic bilayer deformations and harmonic local thickness fluctuations, Landau-de Gennes theory predicts an exponentially declining hydrophobic membrane width between the peptide and the bilayer at infinite distance from the peptide. ${ }^{21}$ Figure $4 \mathrm{C}$ shows that both peptides induce exponential variations consistent with this model (see Materials and Methods). The relative free energy contribution associated with the hydrophobic mismatch-induced deformation estimated from the fit was found to be $\sim 3$ times higher for W16, which has a 10-residue hydrophobic core and shows strong "negative mismatch", compared to W23, whose 17-residue hydrophobic core produces only a slight positive mismatch. The value is on the same order of magnitude as a recent study on bilayer distortion properties of peptides with varying polyleucine core lengths, which suggests relative free energy increases of 2-5 times when going from a 17-residue to a 10-residue hydrophobic core. ${ }^{39}$

Estimating the Insertion Energetics. How favorable is TM insertion from the intermediate SB state? Estimates of partition-

(37) Hristova, K.; Wimley, W. C.; Mishra, V. K.; Anantharamiah, G. M.; Segrest, J. P.; White, S. H. J. Mol. Biol. 1999, 290 (1), 99-117.

(38) Hristova, K.; Dempsey, C. E.; White, S. H. Biophys. J. 2001, 80 (2), 801-11.

(39) Jaud, S.; Fernandez-Vidal, M.; Nilsson, I.; Meindl-Beinker, N. M.; Hubner, N. C.; Tobias, D. J.; von Heijne, G.; White, S. H. Proc. Natl. Acad. Sci. U.S.A. 2009, 106 (28), 11588-93. 

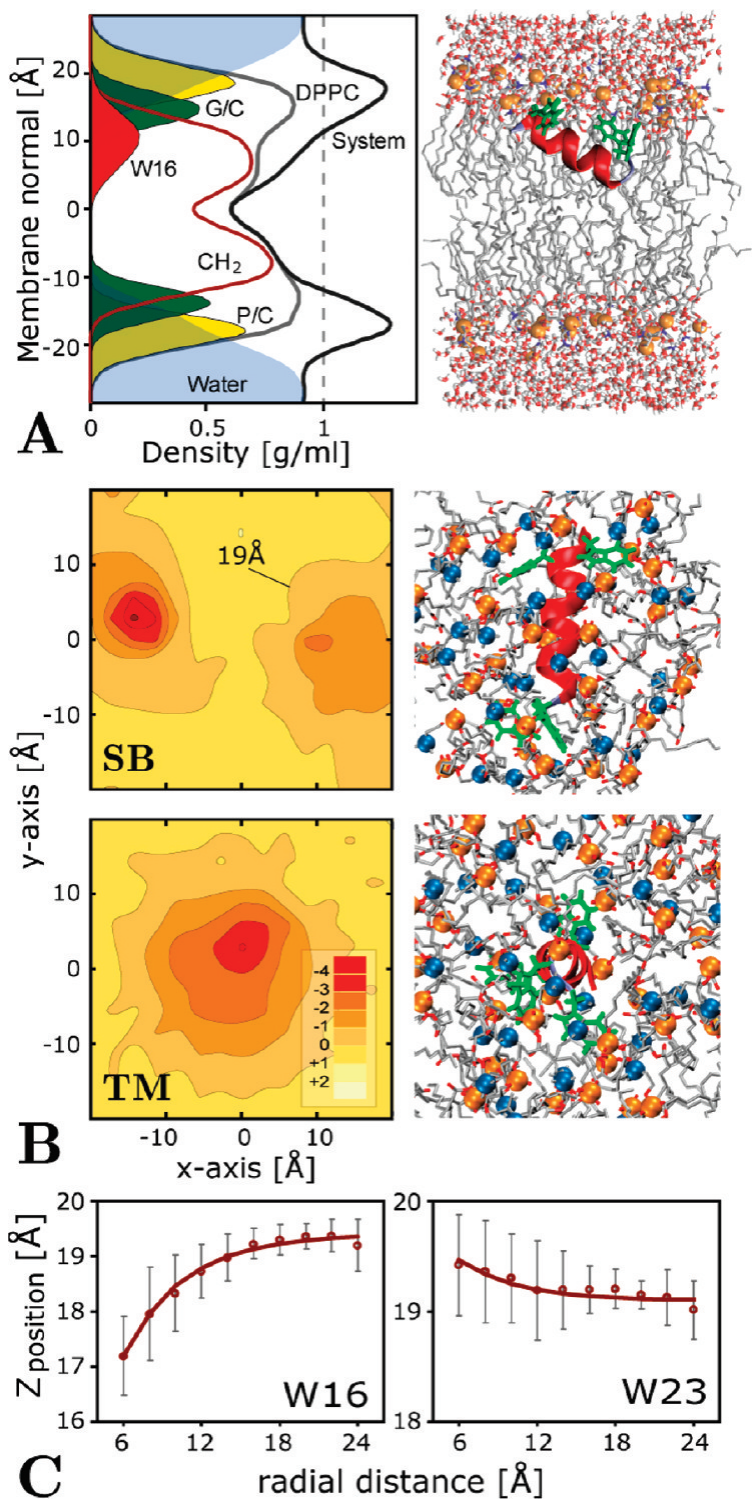

Figure 4. (A) A density cross-section of the bilayer shows that the surfacebound equilibrium configuration $(t=0.8-1.0 \mu \mathrm{s})$ of W16 is located just below the water interface. A representative conformer is shown to scale. Contacts are chiefly with the alkane tails $\left(\mathrm{CH}_{2}\right)$, and there is only a small overlap with the phosphocholine $(\mathrm{P} / \mathrm{C})$ headgroups and carbonyl-glycerol (C/G) groups. (B) The equilibrium phase time-averaged phosphate position from the bilayer center for the surface-bound (SB) and membrane-spanning (TM) helix shows the peptide-induced distortion to the bilayer. Representative conformers are indicated, showing that the P/C headgroups cover the peptide in both configurations (the nitrogen atom of choline is represented as a blue sphere, and the phosphor atom of the phosphate is orange). Results for W23 are similar (Figure 5). (C) For the TM configuration the timeaveraged phosphate position along the membrane normal $\left(Z_{\text {position }}\right)$ varies exponentially with radial distance from the peptide. For negatively mismatched W16, this results in a significant local decrease in bilayer width around the peptide, while W23 shows a slight positive mismatch.

ing free energies for spontaneously inserting peptides range from 5 to $15 \mathrm{kcal} / \mathrm{mol}$, depending on the hydrophobicity. ${ }^{4,32,40-43}$ This

(40) Soekarjo, M.; Eisenhawer, M.; Kuhn, A.; Vogel, H. Biochemistry 1996, 35 (4), 1232-1241.

(41) Bechinger, B. J. Mol. Biol. 1996, 263 (5), 768-775.

(42) Hunt, J. F.; Rath, P.; Rothschild, K. J.; Engelman, D. M. Biochemistry 1997, 36 (49), 15177-15192.

(43) Ulmschneider, M. B.; Sansom, M. S.; Di Nola, A. Biophys. J. 2006, 90 (5), 1650-1660.
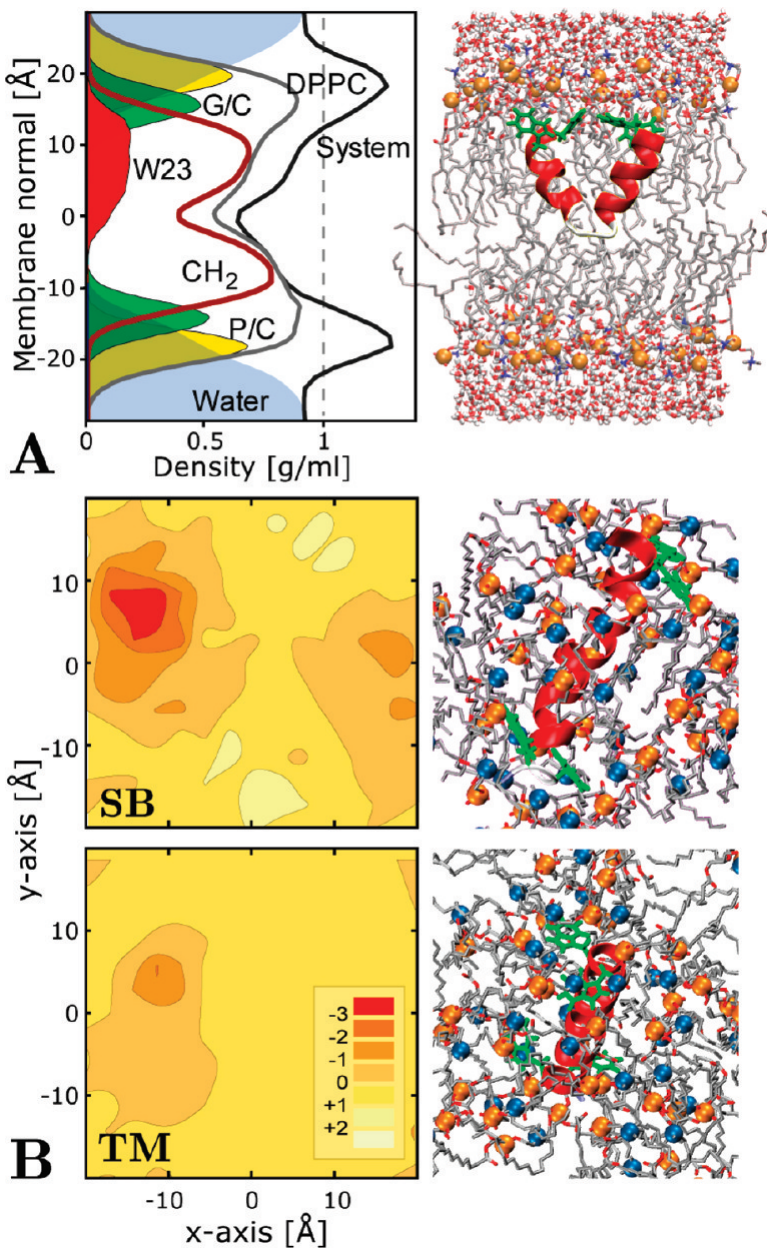

Figure 5. (A) Density profile of the surface-bound W23 peptide in a DPPC lipid bilayer. A representative snapshot of simulation is shown to scale. (B) Bilayer surface distortion induced by the W23 peptide. The contour graph shows the rotationally adjusted deviation in time-averaged phosphate distance from the center of the bilayer. The surface-bound peptide (SB) requires the headgroups to bend over the length of the peptide. This results in a decrease in bilayer density around the flanks of the helix. The transmembrane (TM) orientation displays near-perfect matching, resulting in only minor distortions to the bilayer surface.

means that non-TM states have populations $<0.1 \%$, making them difficult to detect experimentally. Computationally, thermodynamic data can usually be obtained using replica exchange simulations (REMD). As shown in Figure 2, at higher temperature insertion is accelerated. We therefore performed a $200 \mathrm{~ns}$ REMD simulation of W16 starting in the SB state with 32 exponentially spaced replicas over the temperature range $58-225^{\circ} \mathrm{C} ; 225^{\circ} \mathrm{C}$ represents the defacto upper bound, as the bilayer disintegrates if heated further. Interestingly, the peptides remain predominantly helical even in these hot conditions, with only $\sim 50 \%$ loss of helical content at $225{ }^{\circ} \mathrm{C}$ for W16 (Figure $3)$. This remarkable thermostability was further investigated experimentally below.

During the simulation any barrier to insertion is rapidly overcome, with the fraction of replicas that have inserted at least once approaching $100 \%$ within 50 ns (Figure 3, inset). Correspondingly, there is a steady decline of the SB population, and after $\sim 180 \mathrm{~ns}$ all replicas are TM. Insertion is observed to be a one-way process, with no transitions back to the membrane interface at any time. The peptide cannot be dislodged from the TM state by heating, as bilayer disintegration precedes expulsion. The absence of the SB state in the equilibrium phase 
Table 2. List of Simulations Performed ${ }^{a}$

\begin{tabular}{llllll}
\hline peptide & system & \multicolumn{1}{c}{ start $^{b}$} & \multicolumn{1}{c}{$T\left({ }^{\circ} \mathrm{C}\right)$} & \multicolumn{1}{c}{ duration $(\mathrm{ns})$} & \multicolumn{1}{c}{$t_{\text {tolding }}(\mathrm{ns})$} \\
\hline W16 & DMPC & M & 50 & 1000 & 600 \\
W16 & DPPC & M & 80 & 1000 & 150 \\
W16 & DPPC & S & 80 & $2 \times 1000$ & 200,970 \\
W16 & DPPC & S (helix) & $58-225$ & $32 \times 200^{c}$ & - \\
W16 & DPPC & S & $158-225$ & $36 \times \sim 200^{d}$ & see the text \\
W16 & TIP3P & helix & 27 & 500 & $<100^{e}$ \\
W16 & TIP3P & helix & 50 & 500 & $<100^{e}$ \\
W23 & DPPC & M & 80 & 2000 & 140 \\
W23 & DPPC & S & 80 & 1000,1400 & 240,100 \\
W23 & DPPC & S & $175-225$ & $36 \times \sim 200^{d}$ & see text \\
5L & DPPC & S & 80 & 1000 & 180 \\
5L & DPPC & S & $80-160$ & $36 \times \sim 200^{d}$ & see the text
\end{tabular}

${ }^{a}$ The total simulation time was $\sim 35 \mu \mathrm{s} .{ }^{b}$ Starting configuration: M $=$ membrane spanning extended, $\mathrm{S}=$ solvent extended, helix $=$ helical. ${ }^{c}$ Replica exchange simulation with 32 replicas, each replica was run for 200 ns. ${ }^{d}$ Arrhenius simulations, 6-10 simulations at 4-6 temperatures for $15-1000 \mathrm{~ns}$ (average of $\sim 200 \mathrm{~ns}$ ). ${ }^{e}$ Unfolding time (to reach less than $20 \%$ helicity).

( $>180 \mathrm{~ns}$ ) prevents quantification of $\Delta G_{\mathrm{SB} \rightarrow \mathrm{TM}}$ by means of a population analysis, but indicates that the value must be very negative. $\Delta H_{\mathrm{SB} \rightarrow \mathrm{TM}}$ can be calculated directly from the system potential energy, although the fluctuations (estimated from block averaging) are significant, necessitating long simulations (Table 2). For $\mathrm{W} 16$ at $80{ }^{\circ} \mathrm{C}$, we obtain $\Delta H_{\mathrm{SB} \rightarrow \mathrm{TM}}=-0.8 \pm 0.3 \mathrm{kcal} /$ mol per residue. At higher temperatures of $158-212{ }^{\circ} \mathrm{C}$, $\Delta H_{\mathrm{SB} \rightarrow \mathrm{TM}}$ is in a similar range of $-0.8-1.3 \pm 0.5 \mathrm{kcal} / \mathrm{mol}$. For W23, we obtain $\Delta H_{\mathrm{SB} \rightarrow \mathrm{TM}}=-1.0 \pm 0.3 \mathrm{kcal} / \mathrm{mol}$ per residue. A larger insertion is observed for the $5 \mathrm{~L}$ peptide, with $\Delta H_{\mathrm{SB} \rightarrow \mathrm{TM}}=-2.0 \pm 0.8 \mathrm{kcal} / \mathrm{mol}$ per residue, but the errors here are significant due to the short lifetime of the interfacial state. In summary, TM states are strongly favored over SB.

Determination of the Insertion Barrier. The kinetics of the insertion process is difficult to estimate at room temperature or even at $50{ }^{\circ} \mathrm{C}$ due to the limited time scale of $\sim 1-2 \mu \mathrm{s}$ achievable in the MD simulations. Similar to the REMD simulations, insertion can be accelerated by raising the temperature (see Figure 3). Due to the thermostability of the peptides, no folding/unfolding events complicate the kinetic scheme, which corresponds to a simple two-state partitioning process of a rigid $\alpha$-helix. Starting from the interfacial helix of the $80{ }^{\circ} \mathrm{C}$ simulation, 6-10 simulations were performed each for a series of temperatures ranging from 80 to $225^{\circ} \mathrm{C}$, and the time taken to insertion was measured. Arrhenius plots of the logarithm of the insertion rate $k$ as a function of $1 / T$ are shown in Figure 6. In all cases, an excellent fit of $k \sim \exp \left(-\beta \Delta H^{\ddagger}\right)$ can be achieved (quality of fit $r^{2}>98 \%$ ), indicating a firstorder, single barrier process. For this, $k$ can be expressed as

$$
\ln k=\ln A+\frac{\Delta S_{\mathrm{in}}^{\ddagger}}{R}-\frac{\Delta H_{\mathrm{in}}^{\ddagger}}{R T}
$$

where $\Delta H_{\text {in }}{ }^{\ddagger}$ and $\Delta S_{\text {in }}{ }^{\ddagger}$ are the activation enthalpy and entropy of insertion, respectively, and $\ln A$ is a pre-exponential factor. $\Delta H_{\text {in }}{ }^{\ddagger}$ can be extracted straightforwardly from the slope of the Arrhenius plot. However, $\Delta S_{\text {in }}{ }^{\ddagger}$ and the activation free energy $\Delta G_{\text {in }}{ }^{\ddagger}=\Delta H_{\text {in }}{ }^{\ddagger}-T \Delta S_{\text {in }}{ }^{\ddagger}$ can only be obtained by using a specific reaction model that provides an estimate of $\ln A$. Similar to the approach of Yano and Matsuzaki, ${ }^{44}$ we use a theoretical model originally developed to describe the dissociation of a detergent

(44) Yano, Y.; Matsuzaki, K. Biochemistry 2002, 41 (41), 12407-12413.
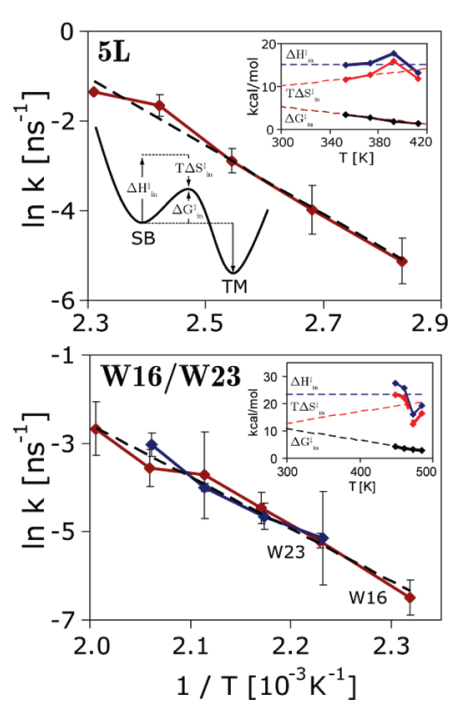

Figure 6. Arrhenius plots of the peptide $\mathrm{SB} \rightarrow \mathrm{TM}$ insertion rate. All peptides exhibit single exponential kinetics with a barrier height of $\Delta H_{\text {in }}{ }^{7}$ $=15 \mathrm{kcal} / \mathrm{mol}$ for $5 \mathrm{~L}$ and $\sim 23 \mathrm{kcal} / \mathrm{mol}$ for both W16 and W23. Error bars are derived from block averages. (Inset) Enthalpic and entropic contributions obtained by fitting individual segments, with dashed lines from overall fit.

monomer from micelles ${ }^{45}$ or a lipid molecule from DOPC vesicles. ${ }^{46}$ In this model, $A=D_{\mathrm{m}} / l_{\mathrm{b}}{ }^{2}$, where $D_{\mathrm{m}}$ is the diffusion constant of the exiting monomer, and $l_{\mathrm{b}}$ is the width of the barrier $R T$ energy units below its maximum. $A$ can be seen as the rate of diffusional motion over the distance $l_{\mathrm{b}}$. Yano et al. have used the lateral diffusion constant of rhodopsin $D_{\text {lat }}=1.8$ $\times 10^{-8} \mathrm{~cm}^{2} / \mathrm{s}$ as an approximation of $D_{\mathrm{m}} \cdot{ }^{44}$ However, the smaller peptides studied here diffuse much more rapidly. Pampel et al. report $D_{\text {lat }}=7 \times 10^{-8} \mathrm{~cm}^{2} / \mathrm{s}$ for W16 from pulsed field gradient NMR in POPC bilayers at $37^{\circ} \mathrm{C},{ }^{47}$ which is almost as fast as the self-diffusion of the lipid molecules at $D_{\text {lat }}=11.1 \times 10^{-8}$ $\mathrm{cm}^{2} / \mathrm{s}$. $l_{\mathrm{b}}$ has been approximated as $1 \AA .{ }^{44}$ Using the diffusion value for $\mathrm{W} 16$, the pre-exponential factor is estimated as $A=$ $0.7 \mathrm{~ns}^{-1}$.

Fitting to the slope of the Arrhenius plots results in $\Delta H_{\text {in }}{ }^{\ddagger}=$ $15.1 \pm 2 \mathrm{kcal} / \mathrm{mol}$ for the $5 \mathrm{~L}$ peptide (Table 1 ). Significantly larger values are obtained for the WALP peptides, with $\Delta H_{\text {in }}{ }^{\ddagger}$ $=23.3 \pm 5 \mathrm{kcal} / \mathrm{mol}$ for $\mathrm{W} 16$ and $\Delta H_{\text {in }}{ }^{\ddagger}=24.2 \pm 6 \mathrm{kcal} / \mathrm{mol}$ for W23. Extrapolated to room temperature $\left(25^{\circ} \mathrm{C}\right)$, the insertion times are $\tau=8.5 \pm 3 \mu$ s for $5 \mathrm{~L}, \tau=107 \pm 15 \mathrm{~ms}$ for $\mathrm{W} 16$, and $\tau=163 \pm 24 \mathrm{~ms}$ for W23 (Table 1), which is beyond the time scales typically achievable in MD simulations. At $80^{\circ} \mathrm{C}$, sampling is $\sim 500$ times faster for WALP and $\sim 50$ times for 5L. Clearly, purely hydrophobic peptides such as $5 \mathrm{~L}$ insert rapidly. For the WALP peptides, Trp translocation seems to be the rate-limiting step, as the insertion rate is similar for the longer peptide. This process is much slower, with a $\sim 8 \mathrm{kcal} /$ mol higher barrier than for 5L. Interestingly, $\Delta H_{\text {in }}{ }^{\ddagger}$ does not exhibit a significant explicit temperature dependence (Figure 6 , insets), indicating that the bilayer properties (i.e., hydrophobicity profile) do not change much upon heating. The kinetic model predicts significant entropic $\left(T \Delta S_{\text {in }}{ }^{\ddagger}\right)$ compensation of up to $\sim 50-60 \%$ of $\Delta H_{\text {in }}{ }^{\ddagger}$ (Table 1 ). For phospholipids, this

(45) Aniansson, E. A. G.; Wall, S. N.; Almgren, M.; Hoffmann, H.; Kielmann, I.; Ulbricht, W.; Zana, R.; Lang, J.; Tondre, C. J. Phys. Chem. 1976, 80 (9), 905-922.

(46) Nichols, J. W. Biochemistry 1985, 24 (23), 6390-6398.

(47) Pampel, A.; Karger, J.; Michel, D. Chem. Phys. Lett. 2003, 379, 555561. 
Table 3. List of Circular Dichroism (CD) Experiments

\begin{tabular}{clccc}
\hline peptide $^{a}$ & \multicolumn{1}{c}{ solvent $^{b}$} & P/L & temp range $^{c}\left({ }^{\circ} \mathrm{C}\right)$ & melting $^{d}(\%)$ \\
\hline W16 & DMPC LUVs & $1 / 100$ & $25-90$ & $1.6 \pm 1.3$ \\
W16 & DPPC LUVs & $1 / 100$ & $45-90$ & $5.4 \pm 1.6$ \\
W16 & DPPC LUVs & $1 / 50$ & $45-90$ & $3.3 \pm 1.0$ \\
W16 & DOPC LUVs & $1 / 100$ & $10-90$ & $2.0 \pm 2.9$ \\
W16 & 1-octanol & - & $20-90$ & $5.2 \pm 1.9$ \\
W16 & water & - & $10-90$ & no signal \\
W23 & DMPC LUVs & $1 / 100$ & $25-90$ & $1.7 \pm 1.5$ \\
W23 & DPPC LUVs & $1 / 100$ & $45-90$ & $1.4 \pm 1.5$ \\
W23 & DPPC LUVs & $1 / 50$ & $45-90$ & $0.8 \pm 0.9$ \\
W23 & DPPC LUVs & $1 / 25$ & $45-90$ & $0.3 \pm 0.1$ \\
W23 & 1-octanol & - & $20-90$ & $2.2 \pm 1.4$ \\
W23 & water & - & $10-90$ & no signal \\
& & & & \\
\hline
\end{tabular}

${ }^{a}$ Peptide concentration was $30 \mu \mathrm{M}$ for all samples. ${ }^{b}$ LUVs $=$ large unilamellar vesicles. ${ }^{c}$ Temperature ranges were sampled in $5^{\circ}$ steps and are only reported above the liquid phase transitions (phase transitions are DMPC, $\sim 23{ }^{\circ} \mathrm{C}$; DPPC, $\sim 42{ }^{\circ} \mathrm{C}$; DOPC, $\sim-20{ }^{\circ} \mathrm{C}$; 1-octanol, $\left.\sim-16{ }^{\circ} \mathrm{C}\right) .{ }^{d}$ Melting was quantified using CDSTR and CONTINLL and averaging the results. Due to the noise in the CD signal below $200 \mathrm{~nm}$, the helicity was quantified for a reduced spectral range above $200 \mathrm{~nm}$ for DOPC and 1-octanol.

has been found to be as large as $\sim 38 \%,{ }^{46}$ while almost entirely enthalpic barriers are reported for the dissociation of lipids ${ }^{46}$ or peptides ${ }^{44}$ from the bilayer. As a result, the free energy barriers are $\Delta G_{\text {in }}{ }^{\ddagger} \sim 5 \mathrm{kcal} / \mathrm{mol}$ for $5 \mathrm{~L}$ and $\sim 11 \mathrm{kcal} / \mathrm{mol}$ for W16 and W23.

Thermostability. The remarkable stability of WALP helices against thermal denaturation was unexpected, as membrane peptides are not generally known to be more stable than soluble proteins. We therefore determined the secondary structure of W16 and W23 using circular dichroism (CD) spectroscopy in DMPC, DPPC, DOPC lipid vesicles and 1-octanol as a function of temperature in the range $10-90{ }^{\circ} \mathrm{C}$ (Table 3). As a control, peptides solubilized in trifluoroethanol (TFE) were added to pure water. Irrespective of temperature, this results in rapid precipitation of aggregates and loss of CD signal, indicating that the peptides are not soluble. Figure 7 shows the spectra and secondary structure analysis for W16 and W23 in DPPC vesicles above the gel-liquid phase transition at $\sim 41{ }^{\circ} \mathrm{C}$. The three distinctive extrema at 208, 222, and $193 \mathrm{~nm}$ are characteristic of an isotropic distribution of $\alpha$-helices, as expected for hydrophobic peptides embedded in the lipid bilayer membrane of vesicles. Secondary structure analysis revealed that both peptides remain helical over the entire temperature range with a loss of helicity of less than $6 \%$ for W16 and no detectable loss for W23. Results in DMPC are similar.

Spectra in DOPC vesicles and 1-octanol show very high noise in the CD signal for wavelengths shorter than $200 \mathrm{~nm}$, making secondary structure determination from the whole spectrum more difficult. However, the $\alpha$-helix content of peptides is directly proportional to the molar residue ellipticity $[\theta]_{\lambda}$ at $\lambda=$ $222 \mathrm{~nm}$, which shows perfect linear behavior against temperature for all environments (Figure 7, insets and Figure 8A), indicating that there is no significant loss of secondary structure. Unfolding of the peptide would be associated with a sigmoidal temperature variation, which is not observed. The slope in DMPC is identical to that of 1-octanol (Figure 8), suggesting that overall hydrophobicity of the environment, rather than entropic contributions due to the lipid tail order, is the chief driver of helical stabilization. Variation of the molar peptide to lipid ratio was found to have no effect on the results, with identical thermostability for ratios in the range from $1 / 100$ to $1 / 25$. The results of the secondary analysis in all environments are summarized
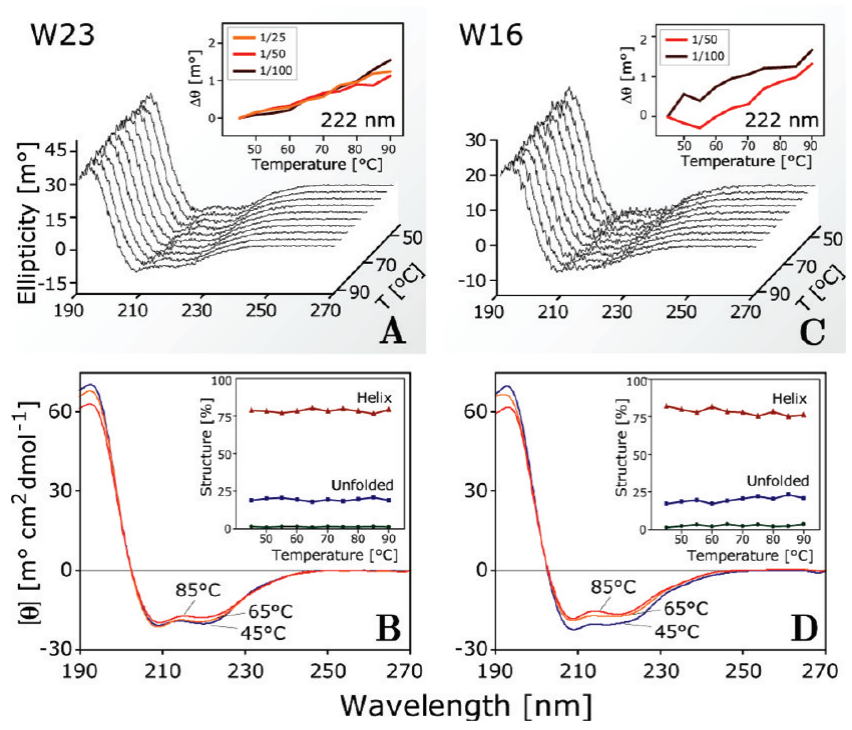

Figure 7. Circular dichroism measurements of the secondary structure of W16 (A, B) and W23 (C, D) in DPPC vesicles (peptide/lipid ratio $=1 / 100$ ) over a temperature range of $45-90{ }^{\circ} \mathrm{C}$. The spectra show three distinctive extrema at 208, 222, and $193 \mathrm{~nm}$, which are characteristic of $\alpha$-helices embedded in the lipid bilayer of vesicles. The $222 \mathrm{~nm}$ line (A, C, insets) shows linear behavior, demonstrating that no melting takes place over the full temperature range. The positions of the peaks in the smoothed spectra show no significant lateral shifts (B, D). Secondary structure analysis (B, $\mathrm{D}$, inset) shows that the peptide remains helical over the entire temperature range with a loss of helicity of less than $6 \%$ for W16 (C) and no detectable loss for W23 (D).

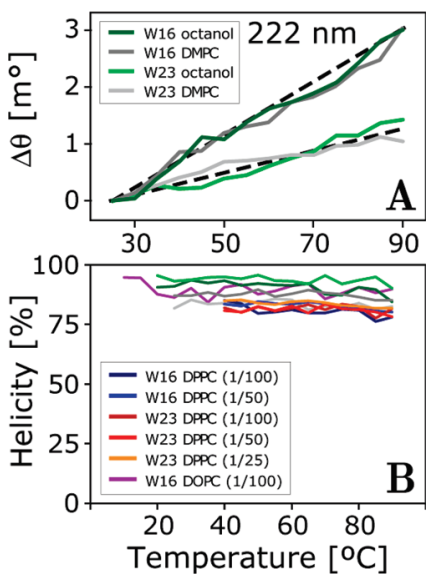

Figure 8. (A) Temperature dependence of the $222 \mathrm{~nm}$ line from the $\mathrm{CD}$ spectra of W16 and W23 in DMPC (peptide to lipid ratio of 1/100) vesicles over a temperature range of $25-90{ }^{\circ} \mathrm{C}$. Perfect linear behavior is observed over the entire temperature range identical to the same peptides in 1-octanol (identical peptide concentration), demonstrating that no melting takes place. (B) Summary of the CD spectra derived helicity versus temperature for W16 and W23 in DMPC, DPPC, DOPC, and 1-octanol. No melting is observed.

in Figure 8B and prove that the thermal stability is a genuine feature of WALP peptides and not a simulation artifact.

\section{Discussion}

Until recently, computational studies of spontaneous peptide partitioning into atomic detail lipid bilayers were thought to be unfeasible due to long simulation time scales required to capture insertion events at physiological temperatures. ${ }^{39}$ Indeed, our estimates for insertion times at room temperature range from 8 $\mu \mathrm{s}$ to $160 \mathrm{~ms}$, above what can be achieved routinely at present. 
However, the present results demonstrate that the partitioning process can be captured in its entirety by simply raising the temperature to $80{ }^{\circ} \mathrm{C}$, where dynamics are orders of magnitude faster (50-500 times). Previously, such heating was thought to lead to denaturation, akin to soluble proteins. Therefore, simulation temperatures above $50{ }^{\circ} \mathrm{C}$ were usually avoided. However, using circular dichroism experiments, we find hydrophobic peptides that serve as model TM helices (W16 and W23) to be extraordinarily thermostable, remaining fully helical even at $90{ }^{\circ} \mathrm{C}$. The thermal stability is observed irrespective of the hydrophobic environment (DMPC, DPPC, DOPC vesicles or 1-octanol) and molar peptide to lipid ratios (1/100 to $1 / 25)$, suggesting that the peptides are stabilized chiefly by desolvation. Burial of backbone hydrogen bonds via helix formation causes a very large energetic stabilization that cannot be overcome through heating. We therefore expect other hydrophobic peptides to show similar resistance against thermal denaturation.

Indeed, the simulations show that the generic hydrophobic peptide $5 \mathrm{~L}$ behaves similar to the tryptophan-flanked WALP peptides. All three peptides remain $80-90 \%$ helical at $80{ }^{\circ} \mathrm{C}$, in agreement with the circular dichroism results. Heating above $225{ }^{\circ} \mathrm{C}$ is required for a $>50 \%$ loss of secondary structure. In contrast, simulations show that these peptides unfold completely in water even at low temperatures (Table 2), proving that the stabilization is due to the hydrophobic bilayer environment rather than being an intrinsic feature of the peptides themselves.

Thermostability is desirable for partitioning studies. Since the bilayer hydrophobicity profile does not change significantly upon heating, the underlying peptide insertion mechanism at elevated temperatures can be assumed to be similar to low-temperature regimes. Indeed, the partitioning pathway (adsorption, interfacial folding, and folded insertion) of W16, W23, and 5L observed in simulations at $80{ }^{\circ} \mathrm{C}$ is consistent with the three-step thermodynamic model of White $\mathrm{e}^{4,48}$ and Engelman. ${ }^{49,50}$ The model predicts that helices must form at the interface prior to insertion, since partitioning an exposed peptide backbone into the hydrophobic bilayer core entails very large energetic penalties, ${ }^{5}$ estimated at $4-5 \mathrm{kcal} / \mathrm{mol}$ per residue. ${ }^{51,52}$ Consistent with this model, all simulations exhibit rapid helical folding upon interfacial adsorption. This "partitioning-folding coupling" 53 is well-known from antimicrobial and synthetic amphiphilic peptides. Bilayer interfaces are potent catalysts of secondary structure formation, ${ }^{54}$ as the cost of moving unmatched peptide bonds to the interface is high $(\sim 1.2 \mathrm{kcal} / \mathrm{mol}) .^{53}$ Hydrogen-bond formation lowers the penalty, ${ }^{55-57}$ driving helix formation at the interface. Experiments on helix-forming amphiphilic peptides give interfacial partitioning-folding free

(48) Jacobs, R. E.; White, S. H. Biochemistry 1989, 28 (8), 3421-3437.

(49) Engelman, D. M.; Chen, Y.; Chin, C. N.; Curran, A. R.; Dixon, A. M.; Dupuy, A. D.; Lee, A. S.; Lehnert, U.; Matthews, E. E.; Reshetnyak, Y. K.; Senes, A.; Popot, J. L. FEBS Lett. 2003, 555 (1), 122-125.

(50) Popot, J. L.; Engelman, D. M. Biochemistry 1990, 29 (17), 40314037.

(51) Roseman, M. A. J. Mol. Biol. 1988, 201 (3), 621-623.

(52) BenTal, N.; Sitkoff, D.; Topol, I. A.; Yang, A. S.; Burt, S. K.; Honig, B. J. Phys. Chem. B 1997, 101 (3), 450-457.

(53) Wimley, W. C.; White, S. H. Nat. Struct. Biol. 1996, 3 (10), $842-$ 848 .

(54) Kaiser, E. T.; Kezdy, F. J. Proc. Natl. Acad. Sci. U.S.A. 1983, 80 (4), 1137-43.

(55) Ladokhin, A. S.; White, S. H. J. Mol. Biol. 1999, 285 (4), 13631369.

(56) Wimley, W. C.; Hristova, K.; Ladokhin, A. S.; Silvestro, L.; Axelsen, P. H.; White, S. H. J. Mol. Biol. 1998, 277 (5), 1091-110.

(57) Fernandez-Vidal, M.; Jayasinghe, S.; Ladokhin, A. S.; White, S. H. J. Mol. Biol. 2007, 370 (3), 459-70. energies in the range $\Delta G_{\mathrm{U} \rightarrow \mathrm{SB}}=-0.1-0.5 \mathrm{kcal} / \mathrm{mol}$ per residue, depending on sequence polarity and hydrophobic moment. ${ }^{57-60}$ Our enthalpic estimates are much more negative $\left(\Delta H_{\mathrm{U} \rightarrow \mathrm{SB}}=\right.$ $-6-10 \mathrm{kcal} / \mathrm{mol}$ per residue) because the completely hydrophobic peptides used in the present study bury deeply into the alkane region of the bilayer (Figures 4 and 5). Amphiphiles instead locate in the semisolvated region of the interface around the height of the glycerol linker. ${ }^{37,38}$

Insertion from the $\mathrm{SB}$ to the native TM configuration is strongly favored, with $\Delta H_{\mathrm{SB} \rightarrow \mathrm{TM}}=-0.8-2.1 \mathrm{kcal} / \mathrm{mol}$ per residue, but there is a high insertion barrier for all systems. The strong partitioning of the peptides studied complicates determination of $\Delta G_{\mathrm{SB} \rightarrow \mathrm{TM}}$, as the $\mathrm{SB}$ state is not sufficiently populated at equilibrium. Experiments have encountered similar problems. Clearly, quantitative estimates of $\Delta G_{\mathrm{SB} \rightarrow \mathrm{TM}}$ will require studying peptides that are less hydrophobic (i.e., insert less strongly).

Curiously, previous explicit simulation studies of W16 partitioning into DPPC bilayers have reported barrierless unfolded insertion pathways ${ }^{11,12}$ and stable unfolded $\mathrm{TM}$ states. $^{12}$ The latter (ref 12) is in direct contradiction to experimental evidence and can be attributed to force field problems, which have since been addressed. ${ }^{13}$ Unfolded insertion in ref 11 was observed using extremely high temperature REMD simulations $\left(77-527^{\circ} \mathrm{C}\right)$, which strongly favors extended conformers. Furthermore, the $\sim 2$ ns simulation time scale of this study was probably too short to reach equilibrium. In comparison, our REMD simulations require $\sim 180$ ns to fully depopulate the SB state (Figure 3D).

An insertion barrier is observed for all three peptides used in this study. Due to their exceptional thermostability, the partitioning kinetics can be derived directly through high-temperature simulations and fitting to an Arrhenius model. Insertion does not involve unfolded conformers but resembles a simple reorientation of a rigid helix from SB to TM configurations, resulting in first-order kinetics. This closely matches timeresolved fluorescence experiments on a related 25-residue hydrophobic peptide into DOPC vesicles performed by Meijberg and Booth, ${ }^{44}$ who report Arrhenius-like kinetics over a temperature range of $20-45^{\circ} \mathrm{C}$, with an estimated activation energy of $\Delta H_{\text {in }}{ }^{\ddagger}=21 \pm 2 \mathrm{kcal} / \mathrm{mol}$. This is remarkably similar to the $15-23 \mathrm{kcal} / \mathrm{mol}$ obtained here. However, insertion at $30{ }^{\circ} \mathrm{C}(\tau$ $=430 \mathrm{~s})$ is orders of magnitude slower than for either WALP $(\sim 0.1 \mathrm{~s})$ or $5 \mathrm{~L}(\sim 10 \mu \mathrm{s})$ in our simulations.

The difference in the barrier heights for the $5 \mathrm{~L}$ and WALP peptides demonstrates that hydrophobic residues contribute much less to the barrier than aromatics like Trp. As insertion of Trp is unfavorable due to its strong interfacial propensity, the insertion barrier for the WALP peptides is dominated by Trp translocation costs. Both $\mathrm{W} 16$ and W23 require $\Delta H_{\text {in }}{ }^{\ddagger} \sim$ $23 \mathrm{kcal} / \mathrm{mol}\left(\Delta G_{\text {in }}{ }^{\ddagger} \sim 11 \mathrm{kcal} / \mathrm{mol}\right)$ to dissociate two adjacent Trp residues from one interface and translocate them across the hydrophobic bilayer core to the other. This compares with potential of mean force (PMF) results of $\sim 1 \mathrm{kcal} / \mathrm{mol}$ from a nonhelical hexapeptide containing one Trp. ${ }^{7}$ Estimates based on Trp scans in designed translocon inserted peptides ${ }^{31,32}$ as well as PMFs derived from statistical analyses of membrane

(58) Li, Y.; Han, X.; Tamm, L. K. Biochemistry 2003, 42 (23), 7245-51. (59) Wieprecht, T.; Apostolov, O.; Beyermann, M.; Seelig, J. J. Mol. Biol. 1999, 294 (3), 785-94.

(60) Ladokhin, A. S.; White, S. H. J. Mol. Biol. 1999, 285 (4), 1363-9. 
protein structures ${ }^{61}$ both show barriers of $\sim 1 \mathrm{kcal} / \mathrm{mol}$ per Trp. PMFs on side-chain analogs give barrier heights of $\sim 0.5-4$ $\mathrm{kcal} / \mathrm{mol}$ per Trp, ${ }^{62,63}$ but none for hydrophobic residues (Ala, Leu), indicating the lack of the polar peptide backbone contributions in these scales.

No dissociation rate could be determined from the present simulations, as this process seems many orders of magnitude slower. Clearly, the very hydrophobic 5L and WALP peptides do not easily dissociate from the membrane, even at very high temperatures, where the bilayer begins to disintegrate. Using fluorescence recovery techniques, Yano et al. have measured a dissociation time of $t_{1 / 2}=10 \mathrm{~h}$ at $25^{\circ} \mathrm{C}$ for a 21 -residue peptide of similar composition, which is $\sim 10$ orders of magnitude slower than what can be reached in simulations. ${ }^{44}$ Thus, unless less hydrophobic peptides are used, it is unlikely dissociation can be simulated directly.

The results in this study demonstrate that the complete peptide partitioning process can now be captured using all-atom

(61) Ulmschneider, M. B.; Sansom, M. S.; Di Nola, A. Proteins: Struct., Funct., Genet. 2005, 59 (2), 252-65. molecular mechanics simulations. The simplicity of this approach suggests a straightforward route to obtaining the dynamics and mechanisms driving spontaneous peptide insertion, providing a powerful tool for systematic exploration and rational design of membrane active peptides. This will greatly improve our understanding of antimicrobial, cell-penetrating, and viral fusion peptides as well as membrane protein stability, assembly, and function.

Acknowledgment. We thank Tania Rutters-Meijneke for help with W16 synthesis. This research was supported by the Human Frontier Science Program (M.B.U.), BIOMS (J.P.U.) and The Netherlands Organization for Scientific Research (NWO-Top grant 700.54.303 to J.P.F.D.). J.C.S. was supported by a Laboratory Directed Research and Development award from the U.S. Department of Energy.

JA909347X

(62) MacCallum, J. L.; Bennett, W. F.; Tieleman, D. P. Biophys. J. 2008, 94 (9), 3393-404.

(63) Johansson, A. C.; Lindahl, E. Proteins 2008, 70 (4), 1332-44. 